\title{
RELATIVISTICALLY PARAMETERIZED EXTENDED HÜCKEL THEORY
}

\author{
L.L. LOHR Jr. \\ Department of Chemistry, Universit 1 of Michigan, \\ Ann Arbor, Afichigan 48109, USA \\ and \\ P. PYYKKÖ \\ Department of Physical Chemustry, Abo Akadem, \\ $S F-20500$ Abo (Turku). Finland
}

Received 3 November 1978; in final form 22 December 1978

\begin{abstract}
A relativistically parameterized extended Hückel molecular orbital method is outlined. One-electron effective hamiltonian matrix elements are related to the corresponding overlap matrix elements calculated in an atomic ( $/$ sjm) basis. Different atomic radial functions are used for the $j=l+\frac{1}{2}$ and $j=l-\frac{l}{2}$ basis functions for a given $n$ and $l$. Relativistic and non-relativistic atomic orbital energies are taken from atomic Dirac-Fock and Hartree-Fock calculations. Results are presented for the molecules $\mathrm{I}_{2}, \mathrm{Br}_{2}, \mathrm{IBr}, \mathrm{HI}, \mathrm{InI}, \mathrm{CH}_{4}, \mathrm{~S}_{1} \mathrm{H}_{4}, \mathrm{GeH}_{4}, \mathrm{SnH}_{4}, \mathrm{PbH}_{4}$, (114)H $\mathrm{H}_{4}$ and $\mathrm{Bl}_{4}$. It is concluded that our method and its parameterization provide a semi-quantitative description of relativ istic effects in chemical bonding-
\end{abstract}

\section{Method}

The basic assumption of a semi-empirical LCAO MO method of the extended Hückel (EHT) type $[1,2]$ is that matrix elements $h_{\bar{i}}$ (for $i \neq j$ ) of an unspecified one-electron effective hamiltonian $h_{\text {eff }}$ are related to the corresponding overlap matrix elements $S_{\hat{i} j}$

$h_{i j}=\left\langle\psi_{i}\left|h_{\mathrm{eff}}\right| \psi_{j}\right\rangle=f\left(h_{i i}, h_{i j}\right) S_{i j}$,

where the function $f\left(h_{i i}, h_{j i}\right)$ is usually expressed in terms of the arithmetic mean of $h_{i i}$ and $h_{i j}$ by

$f\left(h_{i i}, h_{i j}\right)=k\left(h_{i i} \div h_{j i}\right) / 2$,

where the bond constant $k$ is usually assigned a value around 1.75 . Input parameters consist of the molecular geometry, a basis set of atomic orbitals, and a set of atomic orbital energy parameters $\left\{h_{i i}\right\}$ -

In order to obtain semi-quantitative estimates of the bonding properties of molecules containing one or more atoms with large $Z$, and to obtain a measure of the relativistic contribution to such properties, we have developed a relativistically parameterized version of EHT which we designate as "REX". The key fea- tures of REX lie in the choice of atomic basis functions and in the choice of atomic orbital energy parameters. First, the overlap matrix elements $S_{i j}$ are calculated with respect to a set of basis functions with an $\langle\langle\operatorname{sj}\rangle\rangle$ quantization; the procedure for doing this is described in the next section. Second, the [Lsjm functions have associated radial factors obtained by fitting one or more Slater radial functions to the relativistic atomic radial functions of Desclaux [3]. The procedure we have initially used is to choose a single Slater exponent $\zeta$ by matching the electron mean radius $\bar{r}$;

$\zeta=\left(n+\frac{1}{2}\right) / \bar{r}$,

where $n$ is the principal quantum number. The small component of a valence $\mathrm{AO}$ contributes only slightly to $\bar{r}$ and becomes negligibly small in the valence region, compared to the large component. We therefore may relate our two-component Pauli functions directly to Desclaux's four-component $\bar{r}$ values. Since values of $\bar{r}$ are a function of $n, l$, and $j$, the corresponding $\zeta^{\prime} s$ differ for $j=I+\frac{1}{2}$ and $j=l-\frac{1}{2}$ for a given $n$ and $l$. Third, all orbital energy parameters $\left\{h_{i j}\right\}$ are 
taken as the Dirac-Fock orbital energies $\left\{\epsilon_{i}\right\}$. Since Desclaux's tables [3] also include $\bar{r}$ and $\epsilon$ values for the Hartree-Fock non-relativistic limit of $c \rightarrow \infty$, we thus have available consistent relativistic $(c=137.03 \mathrm{au})$ and non-relativistic $(c \rightarrow \infty)$ parameterizations for the $\zeta_{i}$ and $h_{i i}$ values. We denote these two parameterizations as REX and EHT respectively, where both employ the $\mid$ Ljim $\rangle$ basis. Thus direct comparison of REX and the present EHT treatments for a given molecule provides a direct measure of the importance of relativity. Spin-orbit splittings for atomic orbitals with $\boldsymbol{l} \neq \mathbf{0}$ and relativistic shifts for all orbitals. including those with $l=0$, are thus taken into account without a "spin-orbit" hamiltonian being specified. Molecular eiffects enter via eqs. (I) and (2). The eigenvalue problem $(\boldsymbol{H}-\boldsymbol{\epsilon} \mathbf{S}) c=\mathbf{0}$ is then solved by standard methods, taking into account that matrices $H$ and $S$ are in general complex. These matrices are of dimension $2 n \times 2 n$, where $2 n$ is the number of spin-orbitals.

Our method REX resembles that deveioped by Manne, Wittel and Mohanty (hereafter referred to as MWM) $[4]^{\ddagger}$ and applied to a large number of molecules [4-9]. Their interest was primarily in relating MO eigenvaiues to ionization energies obtained by photoelectron spectroscopy. Our method is characterized by a more systematic parameterization, including a well-defined relativistic contraction, or expansion for each orbital. Their calculations were constrained by a common radial factor for the atonic orbitals with $j=l+\frac{1}{2}$ and $j=l-\frac{1}{2}$. Their assumptions [4] about matrix elements (ME's) of an "effective one-electron spin-orbit hamiltonian" $h_{\text {so }}$ include neglect of all three-center and certain two-center ME's, evaluation of onecenter ME's in terms of empirical spin-orbit parameters, and expansion of tw-center ME's involving orbital ea center $A$ connected by the component $h_{\text {so }}$ on center $A$ to an orbital on center $B$ in terms of onecenter (A) ME's.

If the MWM assumptions were applied to our |Lsim $\rangle$ basis, in which the one-center spin-orbit coupling is diagonal, then a two-center $M E$ in a diatomic molecule becomes

$\left\langle\psi_{i}^{\mathrm{A}}\left[h_{s o}\left|\psi_{j}^{\mathrm{B}}\right\rangle=w\left[\left(h_{s \mathrm{O}}^{\mathrm{A}}\right)_{i i}+\left(h_{\mathrm{so}}^{\mathrm{B}}\right)_{j j}\right] s_{i j}\right.\right.$,

where $A$ and $B$ denote the centers, $W V$ is the MWM parameter, and $\left(h_{\text {so }}^{\alpha}\right)_{i i}$ denotes a one-center ME for

This paper contains a thorough discussion of their spinorbit modified extended Hūckel method. center $\alpha$. In REX the corresponding $M E$ is

$\left\langle\psi_{i}^{\mathrm{A}}|\Delta h| \psi_{j}^{\mathrm{B}}\right\rangle=(k / 2)\left(\Delta \epsilon_{i}+\Delta \epsilon_{j}\right) S_{i j}$,

where $\Delta h \equiv h^{\mathrm{R}}-h_{h} \mathrm{NR}$ and $\Delta \epsilon_{i} \equiv \epsilon_{i}^{\mathrm{R}}-\epsilon_{i}^{\mathrm{NR}}$ with $\mathrm{R}$ and NR denoting the relativistic and non-relativistic parameterizations, respectively. The MWM value of $w$ is 0.5 , while $k / 2$ is 0.875 suggesting larger two-center ME's with REX. However the $\left\{\Delta \epsilon_{i}\right\}$ include relativistic shifts as well as splittings.

MWM used atomic spin-orbit splittings and ionization potentials from experimental atomic spectra. Thus their results also include the relativistic shifts. A systematic comparison with non-relativistic results is, however, precluded.

Before the present work was completed, we became aware of the rather analogous, iterative. "self-consistent quasi-relativistic modified extended Hückel" method of Boudreaux et al. [10]. A "quasi-relativistic Mulliken-Wolfsberg-Helmholz" method was also discussed by Bersuker et al. [11], while the entire field of relativistic quantum chemistry has been recently reviewed by Pyykkō [12].

\section{Calculation of overlap matrix}

REX has been written as an adaptation of a standard EHT FORTRAN program ${ }^{*}$. The overlap matrix $S$ in the $\mid l$ sjm $\rangle$ basis is readily calculated by supplying as input data the sets of real atomic orbitals for each atom. The first and second sets consist of those orbitals whose radial functions are later associated with the $j=l-\frac{\pi}{2}$ and $j=l+\frac{1}{2}$ orbitals respectively. Thus for an atom with $s_{2} p_{2}$ and $d A O$ 's, the two sets are associated with $s_{1 / 2}, p_{1 / 2}, d_{3 / 2}$ and $s_{1 / 2}$, $p_{3 / 2}, d_{5 / 2}$, respectively. (The $s A O$ is duplicated so that a generalization to spin-polarized orbitals is possible.) The standard EHT routines for calculating the real overlap matrix in a real $\mathrm{AO}$ basis are employed. One-center overlap matrix elements connecting the two sets are not calculated, as they are not required. $A$ unitary transformation is then used to obtain $S$ in the desired basis. For example, the three real p AO's of the first set are used to construct the two functions

\footnotetext{
The original EHT program was obtained from its author R. Hoffmann in 1966 and is an earlier version of the program recently published [13].
} 
with $j=1 / 2$ and $m= \pm 1 / 2$, while the three real $\mathrm{p}$ AO's of the second set are used to construct the four functions with $j=3 / 2$ and $m= \pm 3 / 2, \pm 1 / 2$. The transformation is based on complex spherical harmonics defined as $[14, p .60]$

$Y_{l m}(\theta, \phi)=N(-1)^{(m+|m|) / 2} P_{l}^{|m|}(\cos \theta) \exp (\mathrm{i} m \phi)$.

where $N$ is a positive normalization constant dependent on $l$ and $m$, and on real harmonics defined as [14, P. 200]

$Z_{l}=Y_{l 0}$,

$Z_{l m}^{\mathrm{e}}=2^{-1 / 2}\left(Y_{l,-m}+\bar{Y}_{l,-m}\right)$,

$Z_{I m}^{\mathrm{s}}=2^{-1 / 2} \mathrm{i}\left(Y_{l_{s}-m}-\bar{Y}_{I,-m}\right)$.

The phase convention for the $\mid l$ sim $\rangle$ basis is expressed in terms of $3-j$ symbols by [15]

$$
|l s j m\rangle=\sum_{m_{l}, m_{s}} C\left(\left[m_{l} \frac{1}{2} m_{s}, j m\right)|| l m_{l} s m_{s}\right)
$$

and

$$
\begin{aligned}
& C\left(m_{l} \frac{1}{2} m_{s} ; j m\right) \\
& \quad=(-1)^{1 / 2-l-m}(2 j+1)^{1 / 2}\left(\begin{array}{llr}
l & \frac{1}{2} & j \\
m_{l} & m_{s}-m
\end{array}\right) .
\end{aligned}
$$

The transformation can be carried out separately for each atom-pair block of the original overlap matrix and separately for $\alpha$ and $\beta$ spins. Thus although formally a $2 n \times 4 n$ transformation matrix is required, the actual transformations used range from $2 \times 2$ for atoms with s AO's only to $32 \times 32$ for atoms with $s$, $p, d$, and f AO's.

\section{Results}

Calculated orbital energies for $I_{2}$ obtained using the parameters in table 1 and a bond length of $2.67 \AA$ are presented in fig. 1 together with experimental values [16-18] and the theoretical values of Manne et al. [4] and of Yang [19], the latter obtained using a Dirac-Slater multiple scattering $\mathrm{X} \alpha$ method. The spin-orbit splitting of the $\pi_{\mathrm{g}}^{*} \mathrm{MO}$ is somewhat less than that of the $\pi_{\mathbf{u}} \mathrm{MO}$, in agreement with experiment. These splittings have been discussed in detail

\begin{tabular}{|c|c|c|c|c|c|}
\hline \multirow[t]{2}{*}{ Element } & \multirow[t]{2}{*}{ Orbital } & \multicolumn{2}{|l|}{$-\alpha_{i}(e V)$} & \multicolumn{2}{|l|}{$\xi_{i}$} \\
\hline & & rel. & non-rel. & rel. & non-rel. \\
\hline H & is & 13.606 & 13.606 & 1.000 & 1.000 \\
\hline$c$ & $\begin{array}{l}2 \mathrm{~s} \\
2 \mathrm{p}_{1 / 2} \\
2 \mathrm{p}_{3 / 2}\end{array}$ & $\begin{array}{l}19.39 \\
11.07 \\
11.06\end{array}$ & $\begin{array}{l}19.38 \\
11.07 \\
11.07\end{array}$ & $\begin{array}{l}1.577 \\
1.435 \\
1.434\end{array}$ & $\begin{array}{l}1.576 \\
1.435 \\
1.435\end{array}$ \\
\hline $\mathbf{S i}$ & $\begin{array}{l}3 s \\
3 p_{1 / 2} \\
3 p_{3 / 2}\end{array}$ & $\begin{array}{r}14.84 \\
7.59 \\
7.56\end{array}$ & $\begin{array}{r}14.79 \\
7.58 \\
7.58\end{array}$ & $\begin{array}{l}1.592 \\
1.257 \\
1.254\end{array}$ & $\begin{array}{l}1.588 \\
1.256 \\
1.256\end{array}$ \\
\hline $\mathrm{Ge}$ & $\begin{array}{l}4 s \\
4 p_{1 / 2} \\
4 p_{3 / 2}\end{array}$ & $\begin{array}{r}15.52 \\
7.42 \\
7.24\end{array}$ & $\begin{array}{r}15.16 \\
7.33 \\
7.33\end{array}$ & $\begin{array}{l}2.057 \\
1.569 \\
1.544\end{array}$ & $\begin{array}{l}2.024 \\
1.550 \\
1.550\end{array}$ \\
\hline Sn & $\begin{array}{l}5 s \\
5 p_{1 / 2} \\
5 p_{3 / 2}\end{array}$ & $\begin{array}{r}13.88 \\
7.01 \\
6.57\end{array}$ & $\begin{array}{r}13.04 \\
6.76 \\
6.76\end{array}$ & $\begin{array}{l}2.218 \\
1.732 \\
1.664\end{array}$ & $\begin{array}{l}2.129 \\
1.674 \\
1.674\end{array}$ \\
\hline $\mathbf{P b}$ & $\begin{array}{l}6 \bar{s} \\
6 p_{1 / 2} \\
6 p_{3 / 2}\end{array}$ & $\begin{array}{r}15.42 \\
7.49 \\
5.98\end{array}$ & $\begin{array}{r}12.49 \\
653 \\
6.53\end{array}$ & $\begin{array}{l}2.718 \\
2.114 \\
1.848\end{array}$ & $\begin{array}{l}2.386 \\
1.880 \\
1.880\end{array}$ \\
\hline 114 & $\begin{array}{l}7 s \\
7 p_{1 / 2} \\
7 p_{3 / 2}\end{array}$ & $\begin{array}{r}19.63 \\
9.67 \\
5.02\end{array}$ & $\begin{array}{l}- \\
-\end{array}$ & $\begin{array}{l}3.405 \\
2.749 \\
1.892\end{array}$ & $\begin{array}{l}- \\
- \\
-\end{array}$ \\
\hline $\mathrm{Br}$ & $\begin{array}{l}4 s \\
4 p_{1 / 2} \\
4 p_{3 / 2}\end{array}$ & $\begin{array}{l}27.78 \\
12.79 \\
12.23\end{array}$ & $\begin{array}{l}27.01 \\
12.44 \\
12.44\end{array}$ & $\begin{array}{l}2.632 \\
2.169 \\
2.124\end{array}$ & $\begin{array}{l}2.588 \\
2.131 \\
2.131\end{array}$ \\
\hline I & $\begin{array}{l}5 s \\
5 p_{1 / 2} \\
5 p_{3 / 2}\end{array}$ & $\begin{array}{l}23.86 \\
11.72 \\
10.58\end{array}$ & $\begin{array}{l}22.34 \\
10.97 \\
10.97\end{array}$ & $\begin{array}{l}2.731 \\
2.289 \\
2.186\end{array}$ & $\begin{array}{l}2.626 \\
2.198 \\
2.198\end{array}$ \\
\hline $\mathrm{Bl}$ & $\begin{array}{l}6 s \\
6 p_{1 / 2} \\
6 p_{3 / 2}\end{array}$ & $\begin{array}{r}18.67 \\
9.21 \\
7.11\end{array}$ & $\begin{array}{r}15.19 \\
7.79 \\
7.79\end{array}$ & $\begin{array}{l}2.898 \\
2.338 \\
2.040\end{array}$ & $\begin{array}{l}2.560 \\
2.072 \\
2.072\end{array}$ \\
\hline
\end{tabular}

Table 1

REX parameters

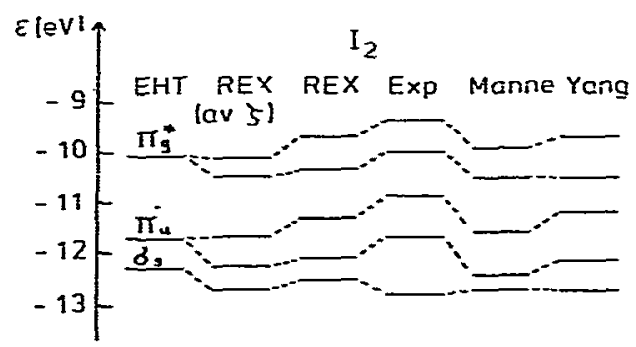

Fig- 1. Calculated non-relativistic (EHT) and relativistic (REX) orbital energies for $I_{2}$. Also show $n$ are the calculated energies of Manne et al. [4] and of Yang [19], and experimental orbital energies $[16 \div 18]$. The results labeled REX (av- 5 ) were obtained using an averaged Slater exponent for both the $5 p_{1 / 2}$ and $5_{P_{3 / 2}} \mathrm{AO}^{\prime} \mathrm{s}$. 


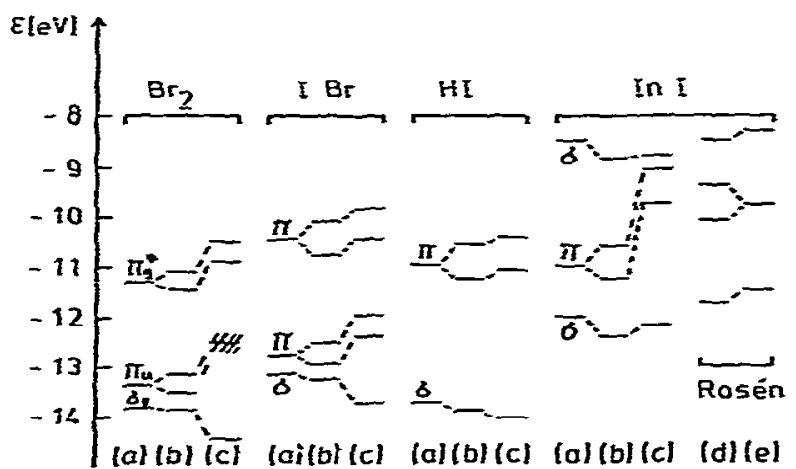

Fio. 2. Calculated non-relativistic (a), relativistic (b), DS DVM (d, ref. [211), and HS DVB1 (e, zef. [21]) orbital erergies together with experimental (c) orbital energies $[16-18,22]$ for $\mathrm{Br}_{2}, \mathrm{IBr}, \mathrm{HI}$, and InI-

by Wittel [5] and by Jungen [20]. We note that use of an averaged Slater exponent $\zeta$ for both the $5 p_{1 / 2}$ and $5 p_{3 / 2}$ AO's produces less satisfactory results than the tse of different $r$ 's. Fig- 2 shows results for $\mathrm{Br}_{2}$, $\mathrm{IBr}, \mathrm{HI}$, and InI, with the latter results compared to those obtained by Rosen [21] using the Dirac-Slater and Hartree-Slater discrete variational methods (DVM). Experimental orbital energies are also shown for $\mathrm{Br}_{2}$ [16-18], H] [16], $1 B r$ [18] and InI [22]- The assumed bond lengths were $2.28,1.61,2.49$, and $2.86 \AA$, respectively. Agreement with experiment is worse for $I B r$ than for eirher $\mathrm{Br}_{2}$ or $\mathrm{I}_{2}$ (fig- 1 ), and is also somewhat unsatisfactory for InI, indicating the shortcomings of a non-charge-iterative method for molecules containing polar bonds.
Table 2 presents our calculated spin-orbit splittings of the valence-shell MO's of symmetry $t_{2}$ for the tetrahedral group IV hydrides. The splitting $\Delta$ is defined as positive when the $u^{\prime}\left(\Gamma_{8}\right.$ or $\left.G_{3 / 2}\right)$ level lies above the $e^{\prime \prime}\left(\Gamma_{7}\right.$ or $\left.E_{5 / 2}\right)$ level. These splittings are compared to the molecular Dirac-Fock onecenter-expansion (DF OCE) $[23,24]$ and atom DiracFock $n$ p splittings [3]. We note that whereas the DF OCE splittings are similar to the corresponding DF central atom splittings, the REX splittings are significantly smaller. This is partly a result of our parameterization (tabie 1), which makes the high $\mathcal{Z}$ elements of this group very electropositive relative to hydrogen, so that an essentially hydridic description obtains, leading to a comparatively small spin-orbit splitting. Since the DF OCE method [23-28] does not in practice allow sufficient "delocalization" we anticipate that actual splittings should fall between the DF OCE and REX limits.

Fig. 3 presents a correlation diagram for the MO's of tetrahedral $\mathrm{Bi}_{4}$ obtained using an assumed $\mathrm{Bi}-\mathrm{Bi}$ distance of $3.0 \mathrm{~A}$. This species has been detected by mass spectrometry in the vapor in equilibrium with the liquid alloy BiSn [29], and is presumably similar to the more familiar species $\mathrm{P}_{4}, \mathrm{As}_{4}$, and $\mathrm{Sb}_{4}$. The highest occupied $M O$ is the relativistic $u^{\prime}\left(\Gamma_{8}\right)$ or the non-relativistic a $\mathrm{MO}$, each at approximately $-8 \mathrm{eV}$. The spin-orbit splitting of the $t_{2}$ lcvels at -8.94 and $-14.43 \mathrm{eV}$ are 1.07 and $0.05 \mathrm{eV}$, respectively, the former splitting being approximately one-half of the atomic $6 \mathrm{p}$ splitting of $2.11 \mathrm{eV}$ (table 1), while the latter is nearly zero because of the predominant $6 \mathrm{~s}$

Table 2

Spin-orbit splittings

\begin{tabular}{|c|c|c|c|c|}
\hline Molecule & $\begin{array}{l}R^{a)} \\
(A)\end{array}$ & $\begin{array}{l}\Delta(\text { REX) } \\
(\mathrm{eV})\end{array}$ & $\begin{array}{l}\left.\Delta(D-F)^{b}\right) \\
(e v)\end{array}$ & $\begin{array}{l}\left.\Delta(\mathrm{D}-\mathrm{F}, \text { atom })^{\mathrm{C}}\right) \\
(\mathrm{eV})\end{array}$ \\
\hline $\mathrm{CH}_{4}$ & 1.095 & 0.0045 & $\begin{array}{l}0.01 \\
0.0056 \text { d) }\end{array}$ & 0.008 \\
\hline $\mathrm{SiH}_{\mathbf{A}}$ & 1.560 & 0.012 & $\begin{array}{l}0.021 \\
0.022 \text { d) }\end{array}$ & 0.03 \\
\hline $\mathrm{GeH}_{4}$ & 1.588 & 0.067 & 0.16 & 0.18 \\
\hline $\mathrm{SnI}_{4}$ & 1.746 & 0.14 & 0.42 & 0.44 \\
\hline $\mathrm{PbH}_{4}$ & 1.746 & 0.49 & 1.54 & 1.50 \\
\hline$(114) \mathrm{H}_{4}$ & 1.746 & 1.68 & 5.16 & 4.66 \\
\hline
\end{tabular}

a) Distances chosen to match those used in refs. $[23,24]$.

b) Refs. [23,241. c) Ref. [3].

d) Includes non-spherical contributions. 


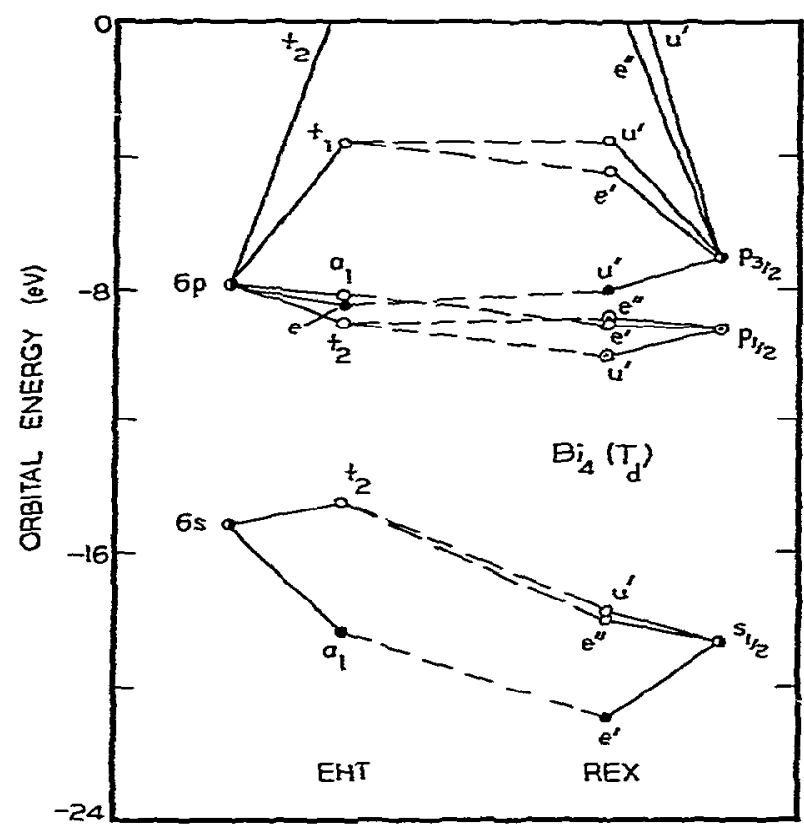

Fig. 3. Calculated non-relativistic (EHT) and relativistic (REX) orbital energy correlation diagram for $\mathrm{Bi}_{4}$ with $\mathrm{T}_{\mathrm{d}}$ symmetry and a $\mathrm{Bi}-\mathrm{Bi}$ distance of $3.0 \mathrm{~A}$.

character of the lower energy $t_{2} \mathrm{MO}$. The icn $\mathrm{Bi}_{4}^{2-}$ would have two electrons in e' $\left(\Gamma_{6}\right.$ or $\left.\mathrm{E}_{1 / 2}\right) \mathrm{MO}$ at $-4.5 \mathrm{eV}$; although a closed-shell system, the gap to the vacant $\mathrm{u}^{\prime}\left(\Gamma_{8}\right) \mathrm{MO}$ is only $0.9 \mathrm{eV}$, so that a secondorder Jahn - Teller instability is expected for tetrahedral $\mathrm{Bi}_{4}^{2}-$ (first-order in the non-relativistic description with two electrons in the $\mathrm{t}_{1} \mathrm{MO}$ ). The ion $\mathrm{Bi}_{4}^{2-}$ is known in the solid state [30] as a planar species of $\mathrm{D}_{4 \mathrm{~h}}$ symmetry with a $\mathrm{Bi}-\mathrm{Bi}$ distance of $2.94 \mathrm{~A}$. Calculations (not shown) for $\mathrm{a}_{4 \mathrm{~h}}$ structure with a $\mathrm{Bi}-\mathrm{Bi}$ distance of $3.0 \AA$ suggest stability for the dianion, with the highest occupied $M O$ being of $e_{g}$ symmetry and lying $1.95 \mathrm{eV}$ below a vacant $\mathrm{b}_{1 \mathrm{u}}$ symmetry MO (the designation with atoms lying between tire $x$ and $y$ axes in the $x y$ plane). This gap is reduced to $1.75 \mathrm{eV}$ when relativistic parameters are used. Similar non-relativistic results were previously reported by Corbett [31].

The tetrahedral $\mathrm{Bi}_{4}$ and the isoelectronic species $\mathrm{Pb}_{4}^{4-}$ are according to fig. 3 stabilized by the large $u^{\prime}-e^{\prime}$ distance. In Lauher's [32] terminology, our $u^{\prime}$ would be the highest "cluster valence molecular or- bital" while the subsequent $e^{\prime}$ is the lowest "high lying anti-bonding orbital". As seen. this division is not essentially changed in the $T_{d}$ case by relativistic effects.

\section{Summary}

Our basic philosophy concerning REX is that it provides a convenient and inexpensive procedure for "extrapolating" the results of Dirac-Fock atomic calculations to molecules, thus providing a semi-quantitative description of relativistic effects in chemical bonding. Comparisons are made between orbital energies and observed ionization energies. The experimental spin-orbit splittings of valence MO's are generally well reproduced by $R E X$, while the absolute $\pi$-MO binding energies tend to be too large. Due to the absence of charge iteration, the hydrides of more electropositive metals tend to be too hydridic. Results for numerous other molecules will appear in a future publication [33].

\section{References}

[1] R. Hoffmann, J. Chem. Phy s. 39 (1963) 1397.

[2] L.I. Lohr Jr. and W.N. Lipseomb, J. Chem. Phys- 38 (1963) 1607.

[3] J.P. Desclaux. At. Data Nucl. Data Tables 12 (1973) 311.

[4] R. Manne, K. Wittel and B.S. Mohaniy, Mol. Phys. 29 (i975) 485.

[5] K. Wittel, Chem. Hhy s. Letters is (1972) 555.

[6] K. Wittel, B.S. Mohanty and R. Manne, J. Electron Spectry. Related Phenomena 5 (1974) 1115.

[7] K. Wittel, H. Bock and R. Manne, Tetrahedron 30 (1974) 651 .

[8] K. Wittel and R. Mtanne, Theoret. Chim. Acta 33 (1974) 347.

[9] K. Wittel and R. Manne, J. Chem. Phys. 63 (1975) 1322

[10] E.A. Boudreaux, T.P. Carsey, A. Dutta-Ahmed, E.S. Elder and L.E. Harris, Abstracts of the EUCHEM Conference on Electronic Structures of Transition Metal Compleves, Maynooth, Ireland (1978).

[11] I B. Bersuker, S.S. Budnikov and B.A. Leizerov, Intern. J. Quantum Chem. 11 (1977) 543.

[12] P. Pyykko, Advan. Quantum Chem. 11 (1978) 353.

[13] J. Howell, A. Rossi, D. Wallace, K. Haraki and R. Hoffmann, QCPE 10 (Suppl.) (1973) 344.

[14] J. S. Griffith, Theory of transition metal ions (Cambridge Univ. Press, London, 1961).

[15] M. Rotenberg, R. Bivins, N. Metropolus and J.K. Wooten Jr., The 3f and 6-j symbols (The Technology Press, MIT, Cambridge, 1959) p. 4. 
[16] D.C. Frost, C.A. McDowell and D.A. Vroom, J. Chem. Phys. 46 (1967) 4255.

[17] A.B. Comford, D.C. Frost, C.A. McDouell, I.L. Ragle and L.A. Stenhouse, J. Chem. Phys 54 (1971) 2651.

[18] A.W. Potts and W.C. Price, Trans. Faraday Soc. 67 (1971) 1242.

[19] C.Y. Yang. Chem. Phys. Letters 41 (1976) 588.

[20] M. Jungen, Theoret. Chim. Acta 27 (1972) 33.

[2I] A. Rosén, Intern. J. Quantum Chem. 13 (1978) 509.

[22] I. Berkow itz and I. E. Dehmer, J. Chem. Phys. 57 (1972) 3194.

[23] J.P. Desclaux and P. Pyykkö, Chem. Phys. Letters 29 (1974) 534

[24] P. Pyykkō and J.P. Desclaux, Nature 226 (1977) 336.
[25] J.P. Desclaux and P. Pyykkō, Chem. Phys. Letters 39 (1976) 300.

[26] P. Pyykkō and S.P. Desclaux, Chem. Phys. Letters 42 (1976) 545.

[27] P. Pyykkō and J.P. Desclaux, Chem. Phys. Letters 50 (1977) 503.

[28] P. Pyykkō and J.P. Desclaux, Chem. Phys. 34 (1978) 261.

[29] G. Riekert, P. Lamparter and S. Steeb, Z. Naturforsch. 31 A (1976) 711.

[30] A. Cisar and J.D. Corbett, Inorg. Chem. 16 (1977) 2482.

[31] J.D. Corbett, Inorg. Nucl. Chem. Letters 5 (1969) 81.

[32] J.W. Lauher, J. Am. Chem. Soc. 100 (1978) 5305.

[33] P. Pyykkō and L_L_ Lohr Jr-, to be pliblished. 